\title{
Central core disease in one of identical twins
}

\author{
M I CHAEL E. COHEN, PATR I C A K. DUFFNER, A N D \\ RE I D H E F F N ER \\ From the Division of Child Neurology and Neuropathology, State University of New York, \\ School of Medicine, Buffalo, New York, USA
}

SUMMARY A case of central core disease in one of identical twins is presented. The light and electron microscopic pathology is reviewed. We believe that these entities are not genetic in this sibship and may be an example of isolated maturational arrest of non-specific aetiology.

Central core disease was first described by Shy and Magee in 1956. The disease was seen in five members from three generations of the same family, suggesting a dominant mode of inheritance. The clinical features are hypotonia, delayed motor milestones, and a mild, non-progressive proximal myopathy. We wish to report a case of central core disease in one of identical twins. To our knowledge this occurrence has not previously been described.

An associated finding in this child is the presence of arthrogryposis multiplex congenita. Although there have been cases of congenital arthrogryposis occurring in either both or one of identical twins (Hillman and Johnson, 1952; Lipton and Morgenstern, 1955) in none of the cases of arthrogryposis multiplex congenita associated with monozygotic twinning have muscle biopsies been done to delineate specific pathology.

This report will document the existence of central core disease in one of monozygotic twins, establish the identical nature of the twinning, and speculate on the aetiology of the arthrogryposis associated with the central core disease.

\section{Case report}

SD, a one year old twin boy, was the product of an eight month pregnancy and breech delivery. $\mathrm{He}$ was the second born of identical twins. There was a single placenta. Birthweight was $1.9 \mathrm{~kg}$ (four pounds, five ounces). Twin A weighed $2 \mathrm{~kg}$ (four pounds, 10 ounces) and was a vertex presentation. After delivery it was noted that the baby was

Address for reprint requests: Michael E. Cohen, MD, Division of Neurology, Buffalo Children's Hospital, 219 Bryant Street, Buffalo, New York 14222, USA.

Accepted 23 February 1978 thinner than twin A and had difficulty in moving his extremities. He had flexion contractures of the elbow and limitation of motion of the shoulders. His feet were in a position of talipes equinovarus. Subsequently, the child was casted and had heel cord lengthening. Currently, the youngster has generalised weakness with limitation of motion at the shoulders and elbows. He is able to move his arms to approximately 100 degrees at the elbow and approximately 90 degrees at the shoulder. His hands are well formed and there are no fixed contractures. He has approximately 60 degrees motion at the ankles. His twin has no evidence of muscle weakness, joint limitations, or hypotonia, and is developmentally normal (Fig. 1). The serum creatine kinase (CPK) was normal. Peroneal, median, and ulnar nerve conduction times were normal. An EMG of the thigh failed to reveal evidence of either myopathy or neuropathy. A muscle biopsy was obtained from the left vastus lateralis muscle under local anaesthesia.

Specimens for routine paraffin sections and electron microscopy were clamped in a Mueller isometric device before fixation in a modified $4 \%$ paraformaldehyde solution (Stefanini et al., 1967). Paraffin sections were stained with haematoxylin and eosin (H and E), periodic acid Schiff (PAS), phosphotungstic acid haematoxylin (PTAH), and Masson's trichrome. Tissue for electron microscopy was post-fixed in osmium tetroxide, dehydrated in graded ethanols, and embedded in Epon 812 . One $\mu \mathrm{m}$ semithin sections were stained with toluidine blue. Ultra-thin sections were prepared using a Reichert OMU-3 ultramicrotome, stained with uranyl acetate and lead citrate, and examined with a Zeiss EM 10 electron microscope.

Tissue for cryostat sections was frozen in liquid nitrogen. Sections were cut on an IEC cryomicrotome and stained with $\mathrm{H}$ and $\mathrm{E}$, modified 


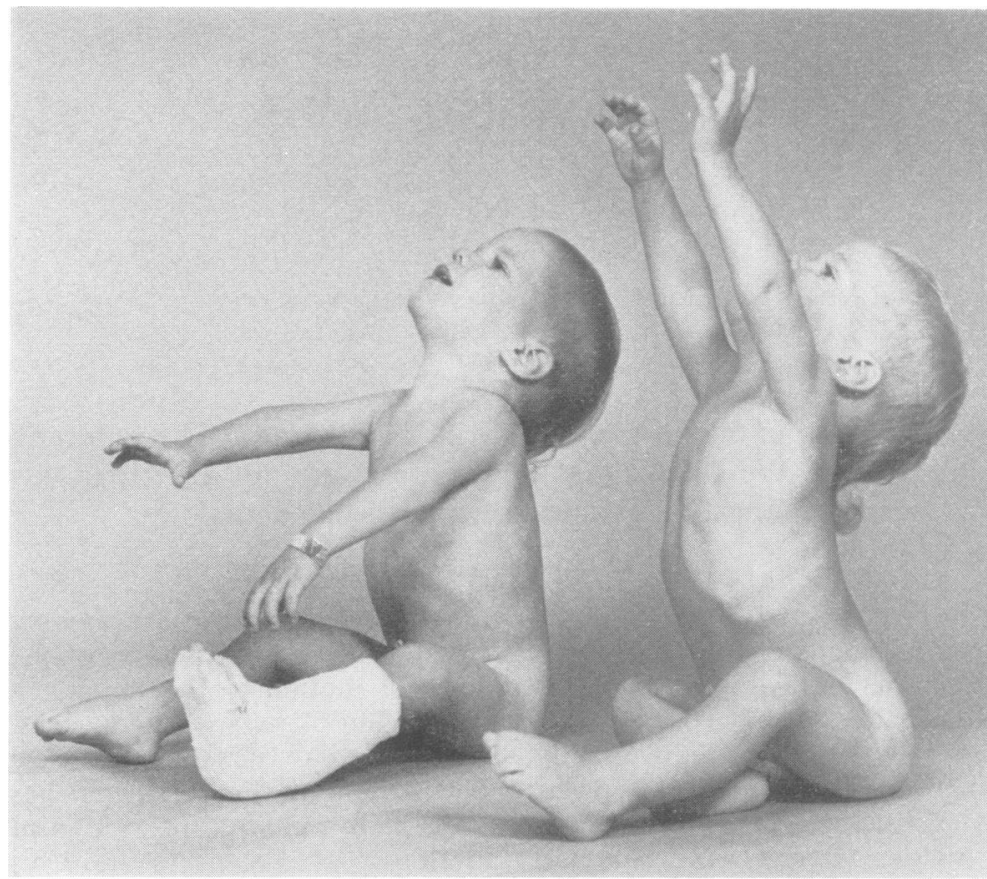

Fig. 1 Photographs of both twins showing limited range of motion of the shoulders in the affected twin.

Gomori trichrome (Engel and Cunningham, 1963), myofibrillar adenosine triphosphatase, $\mathrm{pH} \quad \mathbf{9 \cdot 4}$ (ATPase), reduced nicotinamide adenine dinucleotide-tetrazolium reductase (NADH-TR), non-specific esterase, and phosphorylase. Crosssectional fibre diameters were determined in ATPase-stained sections according to standard methods (Brooke and Engel, 1969a).

Light microscopic examination revealed a wide variation in size of muscle fibres, more apparent in frozen sections. The range of fibre diameters was 2-20 $\mu \mathrm{m}$ with a mean fibre diameter of $12 \mu \mathrm{m}$, somewhat below the mean for this age (Brooke and Engel, 1969b; Aherne et al., 1971). The majority of fibres including those which were atrophic were of round or polygonal shape rather than angular. In ATPase reactions, approximately $90 \%$ of fibres stained as type 1 , indicating marked type 1 predominance. There was no evidence of type-grouping or grouped atrophy. Fibre necrosis, regenerative activity, and interstitial fibrosis were absent. No inflammatory infiltrates in the endomysium or surrounding blood vessels were found.

Approximately $20 \%$ of fibres contained central cores which were single and usually centrallylocated foci or decreased oxidative enzyme activity as seen in NADH-TR preparations (Fig. 2). These foci, measuring from 2 to $8 \mu \mathrm{m}$ in diameter, were arranged with their long axes parallel to the fibre axis. In properly oriented specimens, they ap? peared to extend for long distances, up to $2 \mathrm{~mm}$ in length. Central cores were also visible with ATPase, phosphorylase, and PAS stains in which reduced staining intensity was observed within the cores. In Masson's trichrome stains, cores were demonstrated as amorphous light blue areas while the peripheral normal portions of the fibres assumed the usual brick red colour.

Longitudinal Epon-embedded semithin sections stained with toluidine blue revealed scattered "unstructured" central cores. They were easily identified because of loss of cross striation in the involved areas. Typically cores extended the entire length of the segment of muscle fibre examined. Ultrastructurally, there was an abrupt transition between the core and the neighbouring normal part of the muscle fibre. Within the core disruption of the filament organisation resulted in obliteration of the sarcomere pattern. Myofibrillar disorganisation was accompanied by $\mathrm{Z}$ band streaming and zigzagging (Fig. 3). Few mitochondria and reduced glycogen stores were noted within the cores. Abnormal collections of tubular material, probably of sarcotubular origin, were found occasionally.

The pathology report was felt to be compatible with an anatomical diagnosis of central core disease. 


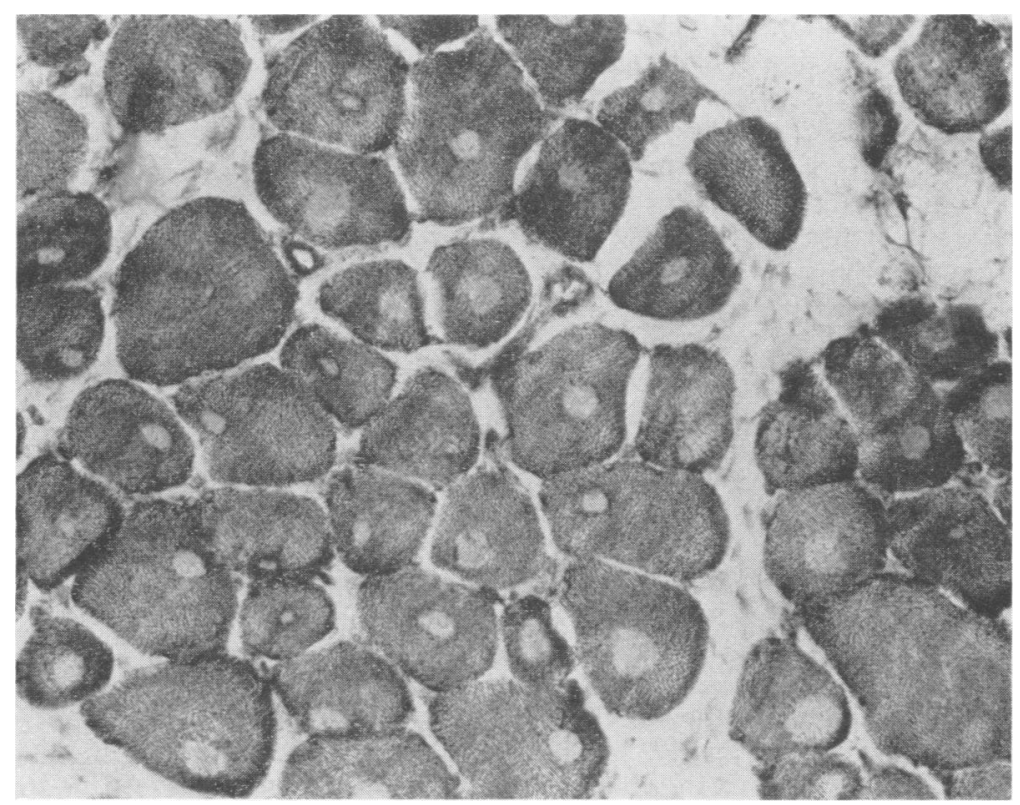

Fig. 2 Numerous central cores with decreased oxidative enzyme activity. NADH-TR, original magnification $\times 200$.

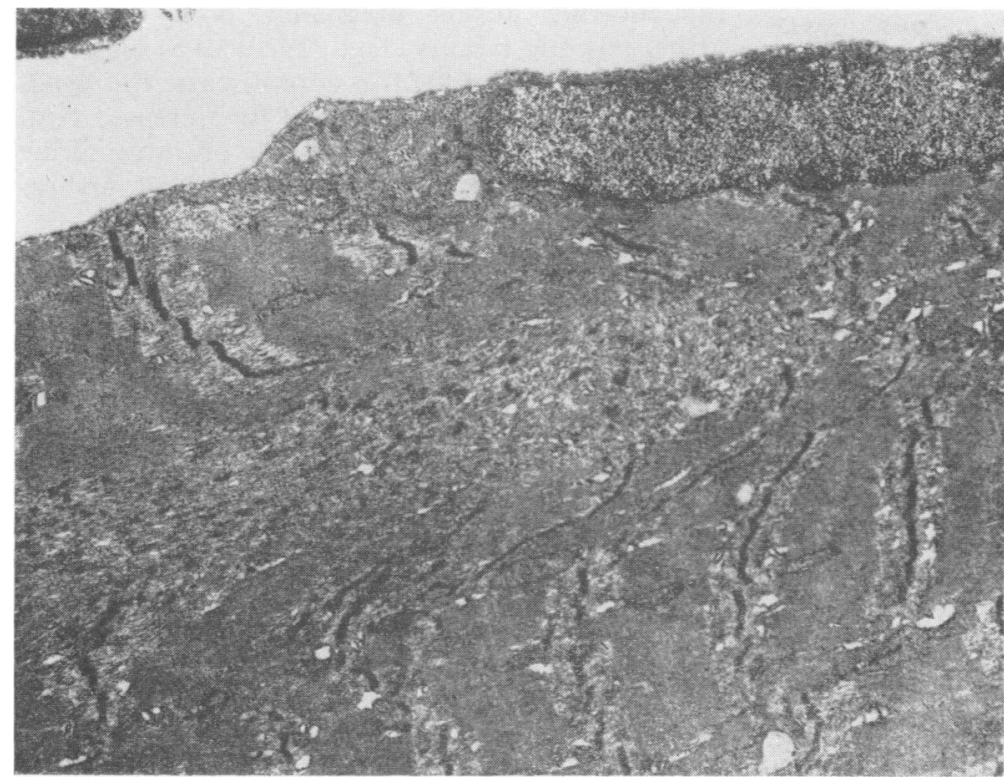

Fig. 3 Electron micrograph of an unstructured core in which myofibrillar disorganisation and $Z$ band streaming is evident. Original magnification $\times 7000$.

\section{EVIDENCE OF MONOZYGOSITY}

The presence of central core disease and arthrogryposis multiplex congenita in one of identical twins raises intriguing possibilities about the aetiologies of these syndromes. The necessity to establish the twin's "monozygosity" is apparent. The twins in this paper had a single placenta. Blood group determinations of each twin revealed both to be $A_{1}$ with the following shared subgroups: $\mathrm{CD} / \mathrm{e}(\mathrm{R} \mathrm{r}) ; \mathrm{NsNs}$; kk; Le(a-b-); Fy $(\mathrm{a}+\mathrm{b}+)$; $\mathrm{Lu}(\mathrm{a}-\mathrm{b}+) ; \mathrm{Jk}(\mathrm{a}+\mathrm{b}+)$.

On HLA typing both twins were noted to share the following four antigens: $A_{1}$, Aw33, B7, Bw35.

The twins have closely associated dermatoglyphics. Both twins had 10 ulnar loops. The total ridge count difference was 12 , which is associated 
with a $3: 1$ chance of monozygosity (Smith and Penrose, 1955). Using the discriminant function developed by Slater, this increases the odds to 20:1 in favour of monozygosity (Slater, 1963).

All this convinced us that the twins were truly monozygotic. The mother's blood type was B+. The father was not available for typing. There is no history either on the maternal or paternal side of muscle weakness.

\section{Discussion}

The genetics of central core disease is poorly understood. Although an autosomal dominant pattern of inheritance has been established in some families, sporadic cases have been reported (Bethlem et al., 1971; Wynne-Davies and LloydRoberts, 1976).

There are several theories regarding the development of central cores. There is evidence that cores are part of the normal maturational stages of fetal muscle. An association with type 1 fibre predominance is suggestive of fetal maturational arrest. Others have suggested that cores are secondary to neuropathic influences and may be a consequence of denervation followed by reinnervation. The similarity in appearance between the target in fibres damaged by denervation and the central core raises the possibility that only a quantitative difference exists between the two. However, not only are central cores more numerous than targets but they are ultrastructurally distinct. The boundary between the core and the normal peripheral portion of the fibre is more abrupt than in the target where a transition zone of less pronounced myofibrillar disruption is observed. Moreover, central cores extend over far greater distances in a longitudinal direction than targets (Heffner, 1975). Hooshmand reported a case of arthrogryposis multiplex congenita with muscle pathology similar to our own, but concluded that his patient had a primary neuropathic disorder with secondary maturational arrest of fetal skeletal muscle. This conclusion was due partly to his finding of an abnormal neuropathic EMG and slowed conduction of nerve (Hooshmand et al., 1971).

In our case, the fact that only one of the identical twins had central core disease indicates that the disease is unrelated to genetic influences. The cores in this case developed after fertilisation and first cellular division. This suggests that environmental influences acting on the developing fetal muscle may have produced this muscle pathology. The normal EMG and nerve conduction times do not support a primary neuropathic aetiology.
The association of central core disease and arthrogryposis multiplex congenita is not unexpected. It has been known that central core disease has been associated with other osteoarticular abnormalities. It has been seen with congenital hip dislocation, pes cavus (Saper and Itabashi, 1976), and clubfoot (Dubowitz and Shavvard, 1968). The central cores in these cases do not appear to be secondary to a previously existing joint abnormality because muscle biopsy samples taken from uninvolved muscle groups-for example, deltoid biopsies in patients with congenital hip dislocations -have shown the central cores (Armstrong et al., 1971). Thus the myopathy appears to be primary. Tellerman-Toppett et al. (1973) have suggested that a muscle biopsy be obtained on all patients with congenital foot deformities even in the absence of weakness, looking for central core disease.

One of the many mechanisms suggested for arthrogryposis multiplex congenita has been the theory of fetal immobility. This has been proposed by Drachman and others (Drachman and Coulombre, 1962). Thus chicks injected with curare in the egg and a mother treated with Dtubocurarine during pregnancy both produced arthrogrypotic infants (Jago, 1970). It is suggested that in the case of central core disease, the hypo- $\mathbb{D}$ tonicity of the infant may lead to decreased fetalo mobility before the third month of intrauterine life and hence produce a syndrome of arthrogryposis multiplex congenita.

Several tentative conclusions may be drawn from this case report. Central core disease in this patient developed from a non-genetic basis. We suggest that in this case, post-fertilisation intrauterine influences may have produced the myopathy. The normal EMG tends to rule against a primary neuropathic factor with a secondary pseudomyopathy as suggested by Hooshmand et al. (1971). Lastly, the central core disease may have caused a relative immobility in early fetal life and, therefore, caused the syndrome of arthrogryposis in our patient. The salient feature in this report is not that the pathological entity is neuropathic or myopathic. We rather suggest that at some point after conception, a skeletal muscle maturational arrest took place. This may have resulted in a pathological picture of central core disease of whatever aetiology leading secondarily to the clinical picture of arthrogryposis multiplex congenita. The finding of osteoarticular abnormalities in isolated joints in other patients with central core disease supports this conclusion.

\section{References}

Aherne, W., Ayyar, D. R., Clarke, P. A., and Walton, 
J. N. (1971). Muscle fibre size in normal infants, children and adolescents. Journal of the Neurological Sciences, 14, 171-182.

Armstrong, R. M., Koenigsberger, R., Mellinger, J., and Lovelace, R. (1971). Central core disease with congenital hip dislocation: study of two families. Neurology (Minneapolis), 21, 369-376.

Bethlem, J., Van Wijngaarden, G. K., Meijer, A. E. F. H., and Fleuky, P. (1971). Observations on central core disease. Journal of the Neurological Sciences, 14, 293-299.

Brooke, M. H., and Engel, W. K. (1969a). The histographic analysis of human muscle biopsies with regard to fiber types. 1. Adult male and female. Neurology (Minneapolis), 19, 221-233.

Brooke, M. H., and Engel, W. K. (1969b). The histographic analysis of human muscle biopsies with regard to fiber types. 4. Children's biopsies. Neurology (Minneapolis), 19, 591-605.

Drachman, D. B., and Coulombre, A. J. (1962). Experimental clubfoot and arthrogryposis multiplex congenita. Lancet, 2, 523-526.

Dubowitz, V., and Shavvard, J. (1968). Congenital clubfoot with questionable central core disease of muscle. Bilateral talipes equinovarus. Proceedings of the Royal Society of Medicine, 61, 1258-1260.

Engel, W. K., and Cunningham, G. C. (1963). Rapid examination of muscle tissue. An improved trichrome method for fresh-frozen biopsy sections. Neurology (Minneapolis), 13, 919-923.

Heffner, R. R. (1975). Electron microscopy of disorders of skeletal muscle. Annals of Clinical Laboratory Science, 5, 338-347.

Hillman, J. W., and Johnson, J. T. H. (1952). Arthrogryposis multiplex congenita in twins. Journal of Bone and Joint Surgery, 34-A, 211-214.
Hooshmand, H., Martinez, A. Julio, and Rosenblum, W. I. (1971). Arthrogryposis multiplex congenita simultaneous involvement of peripheral nerve and skeletal muscle. Archives of Neurology (Chicago), 24, 561-572.

Jago, R. H. (1970). Arthrogryposis following treatment of maternal tetanus with muscle relaxants. Archives of Disease in Childhood, 45, 277-279.

Lipton, E. L., and Morgenstern, S. H. (1955). Arthrogryposis multiplex congenita in identical twins. American Journal of Diseases of Children, 89, 233-236.

Saper, J. R., and Itabashi, H. H. (1976). Central core disease-a congenital myopathy. Diseases of the Nervous System, 37, 649-653.

Shy, G. M., and Magee, K. R. (1956). A new congenital non-progressive myopathy. Brain, 79, 610621.

Slater, E. (1963). Diagnosis of zygosity by fingerprints. Acta Psychiatrica Scandinavica, 39, 28-84.

Smith, M., and Penrose, L. S. (1955). Monozygotic and dizygotic twin diagnosis. Annals of Human Genetics, 19, 273-289.

Stefanini, M., DeMartino, C., and Zamboni, L. (1967). Fixation for ejaculated spermatozoa for electron microscopy. Nature, 216, 173-174.

Tellerman-Toppett, N., Gerard, J. M., and Coërs, C. (1973). Central core disease. A study of clinically unaffected muscle. Journal of the Neurological Sciences, 19, 207-223.

Wynne-Davies, Ruth, and Lloyd-Roberts, G. C. (1976). Arthrogryposis multiplex congenita. Search for prenatal factors in 66 sporadic cases. Archives of Disease in Childhood, 51, 618-623. 